\title{
Psychotropic Use and Sexual Dysfunction: Is There a Practical Solution?
}

\section{Shyamanta Das*}

Department of Psychiatry, Gauhati Medical College Hospital, Guwahati, Assam, India

*Corresponding author: Shyamanta Das, Assistant Professor, Department of Psychiatry, Gauhati Medical College Hospital, Guwahati, Assam, India, Tel: +03612529457; E-mail: dr.shyamantadas@gmail.com

Rec date: July 26, 2017; Acc date: September 18, 2017; Pub date: September 20, 2017

Copyright: (c) 2017 Das S. This is an open-access article distributed under the terms of the creative commons attribution license, which permits unrestricted use, distribution, and reproduction in any medium, provided the original author and source are credited.

\section{Abstract}

The psychotropic medications that we commonly use in our day-to-day life clinical practice are comparable to the players of a football team. If benzodiazepine, whether it is lorazepam, clonazepam, or clobazam is our goalkeeper, then in a 4-3-3 formation we have four strong defenders in the form of fluoxetine, sertraline, paroxetine, and escitalopram. If Lithium is our central defensive midfielder, then the two wingers of our team are valproate and carbamazepine. With quetiapine as our central attacking midfielder, the two lethal strikers at our disposal are risperidone and olanzapine. Thus, we have a good team of 11 players. And we are ready for our game against psychiatric disorders. But, like injury being commonplace for players in a football field, also we have to deal with adverse effects in our practice; sexual dysfunction is one of them. From a category-based classification of psychotropic medications, we are moving towards a mechanism-based one. Talking about mechanisms, prolactin raising 'antipsychotic' is responsible for sexual dysfunction associated with psychotropic use. 'Antidepressant' with higher serotonergic property can also cause sexual dysfunction. There are practical solutions to the problem. Prolactin sparing antipsychotic has less potential to cause sexual dysfunction. Among antidepressants, mirtazapine and bupropion are useful agents. At times, treatment with molecules like sildenafil is necessary. When we combine with this pharmacological approach that of psychosocial intervention, we arrive at a synergic reaction. The ultimate goal is to tailor make our treatment, or in other words 'personalised medicine in psychiatry'.
\end{abstract}

Keywords: Antidepressant; Antipsychotic; Anxiolytic; Anticonvulsant; Psychiatry; Sexual dysfunction; Psychiatric disorders

\section{Introduction}

Since the title of this article is 'Psychotropic use and sexual dysfunction: is there a practical solution?' when we talk of 'psychotropic' and 'practical', they remind us of a football field. A football field comes to our mind when thinking of the common psychotropic drugs that we use in our day-to-day life clinical practice. If benzodiazepine, whether it is lorazepam, clonazepam, or clobazam is our goalkeeper then in a 4-3-3 formation, we have four strong defenders in the form of fluoxetine, sertraline, paroxetine, and escitalopram. If lithium is our central defensive midfielder, then valproate and carbamazepine are our wingers. With quetiapine as the central attacking midfielder, our two strikers are risperidone and olanzapine (Figure 1). Thus, we have a winning team of 11 players. And we are ready for the game against psychiatric disorders. But, an injury in football is commonplace so does we have to deal with adverse effects in our clinical practice. Sexual dysfunction is one such adverse effect.

\section{Interlude}

Traditionally, we are used to clubbing the psychotropic medications into four groups of antipsychotic, antidepressant, anxiolytic, and anticonvulsant. Though certain developments in contemporary psychiatry lead to a paradigm shift in our approach. These developments include Matcheri Keshavan and his team's Bipolar Schizophrenia Network for Intermediate Phenotype, BNIP in short, Thomas Insel and his team's Research Domain Criteria or RDoC,
Stephen Stahl's deconstructing syndrome into symptoms, and Joseph Zohar and his team's Neuroscience-based Nomenclature (NbN).

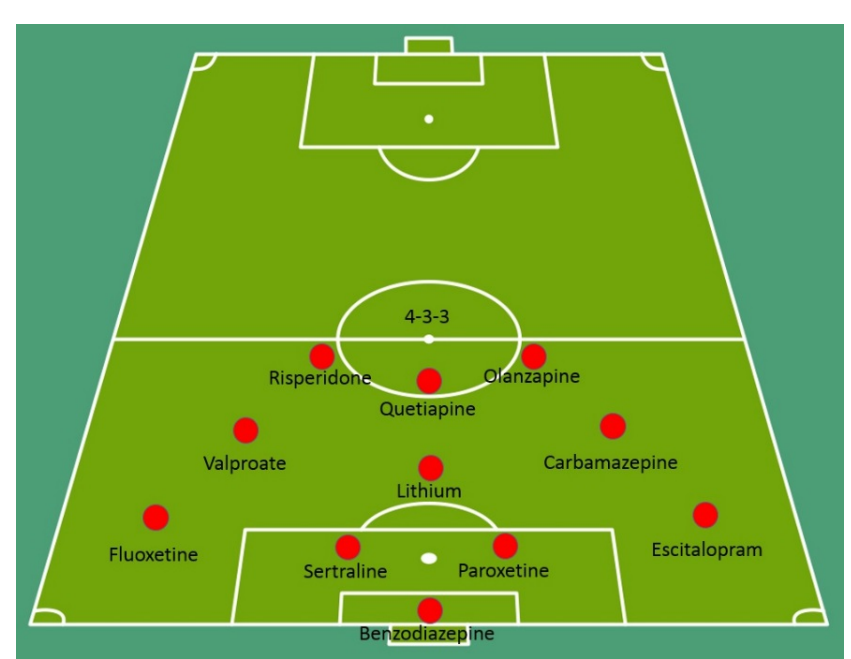

Figure 1: The team.

On one hand, there is an attempt for a brain-based diagnostic system and on the other, the attempt is to have a mechanism-based classification of treatment instead of categories. So, the journey is from phenomenology through neurocircuitry to biological and molecular psychiatry. A corollary of which we can found in oncology.

Our current diagnostic systems of ICD and DSM are comparable to TNM classification, i.e. tumor, node, metastasis. Oncology has moved 
to Human Epidermal Growth Factor Receptor 1 (HER-1) and 2 (HER-2). As is this journey of ours. Thus, a tailor-made treatment or personalized medicine in psychiatry.

If we consider the enlightenment from the middle ages, where mental illness was thought to result from sin and witchcraft (though still present in some parts), as the first revolution in psychiatry, then the second revolution coincides with the evolution of psychoanalysis. The discovery of psychopharmacology dawns the third revolution. The subsequent development in community psychiatry, with emphasis from institutionalization to deinstitutionalization (if we can ignore or incorporate the trans-institutionalization of thereafter), can be identified as the fourth revolution [1].

Now, this common neurobiological underpinning to both diagnosis and therapeutics of psychiatric disorders led us to say that we are entering the fifth revolution of psychiatry [2]. Of course, this is a topic of deliberation in a different platform.

For the discussion of ours here, the traditional approach suffices. But we need to keep this in mind. Though more and more physicians these days are prescribing still the antipsychotic and antidepressant are considered psychiatrists' domains. Thus, 'typical' psychotropic. Anxiolytic and anticonvulsant are used by neurologists for different conditions as much and as long as psychiatrists if not more. So, 'atypical' psychotropic. Let us concentrate on the so-called typical psychotropic here.

\section{Literature Review}

\section{Psychotropic use and sexual dysfunction- part I: Antidepressants}

Sexual behaviour is controlled by the following brain structures: the medial forebrain bundle, the medial preoptic-anterior region of the hypothalamus and its related limbic-hippocampal structures, and the ventral tegmentum of the midbrain [3]. Desire, arousal, and orgasm are the three phases of the human sexual response. This cycle is influenced by several neurotransmitters with different mechanisms [4].

Desire, i.e. the stage one of the sexual cycles is positively influenced by dopamine (DA), melanocortin testosterone, and oestrogen and negatively influenced by prolactin and serotonin (5HT). Erection in men and genital swelling with lubrication in women is the stage two, i.e. arousal that is positively influenced by nitric oxide (NO), norepinephrine (NE), melanocortin, testosterone, acetylcholine (ACh), and DA and negatively influenced by $5 \mathrm{HT}$. Orgasm, i.e. stage three is related with ejaculation in men and is positively influenced by NE (DA and NO have weak positive influence) and negatively influenced by 5HT (Table 1) [4].

\begin{tabular}{|l|l|l|l|}
\hline \multirow{2}{*}{ Neurotransmitters } & \multicolumn{2}{l|}{ Three stages } & \multicolumn{2}{l|}{} \\
\cline { 2 - 4 } & Desire & Arousal & Orgasm \\
\hline Dopamine & + & + & +- \\
\hline Serotonin & - & - & - \\
\hline Norepinephrine & -- & + & + \\
\hline
\end{tabular}

Table 1: Neurotransmitters and sexual cycle.
Sexual inhibitory action characterizes sexual dysfunction associated with antidepressants. Any of the phases in the sexual cycle may be involved alone or together. Commonest sexual dysfunction is delayed ejaculation. Others include problems in orgasm, desire, and/or arousal; erectile dysfunction and less vaginal lubrication included [3].

Serretti and Chiesa [5] recently carried out a meta-analysis. They included studies investigating antidepressant-induced sexual dysfunction. Interestingly these studies used either direct questions or questionnaires. All class of drugs caused sexual dysfunction whether it was selective serotonin reuptake inhibitor (SSRI) (citalopram 79\%, escitalopram $37 \%$, fluoxetine $70 \%$, fluvoxamine $26 \%$, paroxetine $71 \%$, and sertraline $80 \%$ ), serotonin norepinephrine reuptake inhibitor (SNRI) (venlafaxine $80 \%$ and duloxetine $42 \%$ ), tricyclic antidepressant (TCA) (amineptine 7\% and imipramine 44\%), monoamine oxidase inhibitor (MAOI) (phenelzine 42\%), reversible inhibitor of monoamine oxidase A (RIMA) (moclobemide [four per cent]), or others (mirtazapine $24 \%$, bupropion $10 \%$, nefazodone $8 \%$ and agomelatine $4 \%)$. It is important to note that lower sexual dysfunction with fluvoxamine and escitalopram can be the result of the use of less sensitive tools.

Individual antidepressant drug determines its side-effect profile. Prevalence rate varies from ten per cent to $80 \%$ as far as antidepressant-induced sexual dysfunction is concerned, especially SSRIs. Some sexual dysfunction is caused by all antidepressants [3].

\section{It is pertinent to take into account new diagnostic categories:}

- Sexual interest/arousal disorder in women, which includes previous diagnoses of hypoactive sexual desire disorder and female sexual arousal disorder.

- Sexual interest/arousal disorder in men.

- Genito-pelvic pain/penetration disorder, which includes previous diagnoses of vaginismus (not due to a general medical condition) and dyspareunia (not due to a general medical condition).

There are various psychometric rating scales, [3] e.g. Arizona Sexual Experience Scale (ASEX), Changes in Sexual Functioning Questionnaire (CSFQ), Derogatis Sexual Function Inventory (DSFI), Psychotropic-Related Sexual Dysfunction Questionnaire (PRSexDQ), Rush Sexual Inventory (RSI), Sex Effect Scale (Sex FX), Udvalg for Kliniske undersøgelser (UKU).

\section{Treatment of antidepressant-induced sexual dysfunction:}

- Waiting for side effects to reduce spontaneously with time. Reducing drug dose. 'Therapeutic vacation', also called 'drug holiday' whereby in the week drug is reduced or suspended for two days. Switching to bupropion or mirtazapine. Adding symptomatic therapy.

- Symptomatic therapy includes bupropion, nefazodone, mirtazapine, buspirone, cyproheptadine, yohimbine, amantadine, lisuride, bromocriptine, methylphenidate, dextroamphetamine, neostigmine, bethanechol, sildenafil, tadalafil, vardenafil, or herbal medicines.

\section{Psychotropic use and sexual dysfunction- part II: Antipsychotics}

Sexual dysfunction is common with antipsychotic medication. Spontaneous reporting is rare. Direct questioning increases report. Thus, the rate varies depending on the approach. Definitions of sexual dysfunction also determine prevalence rates [6]. 
Research work on the endocrine impact of risperidone shows a high rate of sexual dysfunction [7] that is still less compared to others [8]. Under reporting is a possibility for this discrepancy that again can be due to "lack of extensive questionnaire, culture specific factors, and stigma related issues", as suggested by the authors [7]. In fact, "the frequency of antidepressant-associated sexual dysfunction is greatly underestimated in clinical trials that rely on patient self-report of these adverse events" [9]. What we get as a result is only the tip of the iceberg. "Direct inquiry reveals that delayed orgasm/ejaculation occurs in $>50 \%$ and anorgasmia in at least one-third of patients given selective serotonin reuptake inhibitors" [9].

Binding to dopaminergic, cholinergic, histaminergic and $\alpha$ adrenergic receptors may directly affect sexual function by inhibiting motivation and reward, increasing sedation and reducing peripheral vasodilation [6].

Antipsychotics cause a decrease in DA in the hypothalamic infundibular system leading to hyperprolactinaemia and thus inhibiting sexual function. This elevated prolactin influences gonadotropin releasing hormone $(\mathrm{GnRH})$ in the hypothalamus, thereby affecting follicle stimulating hormone (FSH) and luteinising hormone $(\mathrm{LH})$ in the anterior pituitary, and ultimately reducing oestrogen in women and testosterone in men [6].

First generation antipsychotics, as well as risperidone and amisulpride, increases prolactin leading to the division of antipsychotics as 'prolactin-raising' and 'prolactin-sparing. Prolactinraising antipsychotics cause more sexual side effects than prolactinsparing ones [10].

Though olanzapine is thought to be a prolactin-sparing antipsychotic, we found that in our population it is not so. Both men and women showed an elevation of serum prolactin. What more, women had elevation at a lesser dose and early in treatment compared to men [11].

Antipsychotic-induced side effects on sexual function are usually inhibitory in nature and may affect all phases of the sexual response cycle. These effects include problems with desire, arousal, and/or orgasm [6].

\section{Treatment of sexual dysfunction induced by antipsychotics:}

- Evaluation to rule out other comorbid physical and psychiatric disorders as well as the use of both prescribed and illicit drugs; estimation of prolactin level when hyperprolactinaemia is suspected.

- Risk factor modification in the form of drug use, blood sugar, hypertension, cholesterol. Spontaneous improvement can be awaited in the early phase.

- Reducing dose.

- Switching to prolactin-sparing antipsychotic.

- Therapy targeting symptom like use of amantadine, bromocriptine, cabergoline, phosphodiesterase inhibitors (sildenafil), yohimbine.

\section{Psychotropic use and sexual dysfunction- part III: Mood stabilisers and anxiolytic drugs}

A passing-by comment on the 'atypical' psychotropic. Mood stabilisers do affect sexual function negatively. But, as far as anxiolytics are concerned we do not have clear evidence of an effect on sexual function. The switch of enzyme-inducing anticonvulsant to non- enzyme-inducing ones can help. Treatment is like that used for sexual dysfunction independent of drug use [12].

\section{Discussion}

Sexual dysfunction is a common accompaniment of psychotropic drugs [9]. In the assessment, direct questions instead of specific questionnaires decease correct diagnosis of drug-induced sexual dysfunction [3].

Sexual dysfunction is a side effect of the antidepressant medication that adversely affects desire, arousal, and/or orgasm and sexual dysfunction is highest with more serotonergic properties. Some sexual dysfunction is caused by all antidepressants, notably with SSRIs and SNRIs, in both men and women. However, agomelatine, bupropion, mirtazapine, moclobemide, and nefazodone may not cause an increased sexual side effect. Treatment of benefit includes the addition of more tolerable antidepressants like bupropion and mirtazapine, and phosphodiesterase inhibitors, or drug holidays [3].

All antipsychotics cause sexual dysfunction. Still, the second generation seems better than the first generation as far as the sexual side effect is concerned [6]. Diminished libido is more commonly observed with dopamine antagonist antipsychotics and sexual dysfunction is less with newer atypical antipsychotics with low dopamine effect [9]. Desire, arousal, and/or orgasm alone or in combination are adversely affected by antipsychotic drugs, more so by the 'prolactin-raising' ones [6].

Seizure disorder themselves are associated with sexual dysfunction. Moreover, sex hormones are affected by anticonvulsants. Therefore, baseline sexual function needs to be established [9].

\section{Conclusion}

If we call the act of sex as love making then it is a bridge whose two ends are love and respect. Here two individuals are involved. They are not inanimate. Both have feelings and cognitions. Both have likings and dislikes. Both have dreams and aspirations. Both have their own unique physical structures. In this relationship, are there love and respect? Love and respect to each other's likings and dislikes. Love and respect to each other's dreams and aspirations. Love and respect to each other's physical structures. Love and respect to the soft parts of the body. Love and respect to the hard parts of the body.

Because it is quite often seen that when an individual is diagnosed with a psychiatric disorder she/he is at the receiving end of negative attitude. The same individual who is well-accepted in the society at large when diagnosed with mental illness is at the receiving end of discrimination and prejudice. Is the partner also one of those with such negative attitude? Is this negative attitude affecting the love and respect? As without these two ends of love and respect the bridge of sexual act or making love will be a mismatch.

During the biological evaluation of sexual dysfunction associated with psychotropic use, we need to look into this aspect as well. This combined and comprehensive approach only can guide us in the 'clinical dilemmas in psychiatry' and we can say yes, there is 'a practical solution' to 'psychotropic use and sexual dysfunction'.

\section{References}

1. Ahuja N (2011) A short textbook of psychiatry (7th edn) Jaypee Brothers Medical Publishers, New Delhi, India. 
Citation: Das S (2017) Psychotropic Use and Sexual Dysfunction: Is There a Practical Solution?. J Trauma Treat 6: 394. doi: 10.4172/2167-1222.1000394

Page 4 of 4

2. Das S, Hazarika M, Bardhan N, Talukdar U, Bhagabati D, et al. (2015) Fifth revolution of psychiatry. In: Das S, Medhi D, Dutta J, Chakaravarty $S$, (eds). Brain understanding of mental illness. (1st edn) Academy Publisher, Guwahati, India. pp. 1-10.

3. La Torre A, Giupponi G, Duffy D, Conca A (2013) Sexual dysfunction related to psychotropic drugs: A critical review-part I: Antidepressants. Pharmacopsych 46: 191-199.

4. Stahl SM (2008) Stahl's essential psychopharmacology: Neuroscientific basis and practical applications (3rd edn) Cambridge University Press, UK.

5. Serretti A, Chiesa A (2010) Antidepressants and sexual dysfunction: epidemiology, mechanisms and treatment strategies. Ital J Psychopathol 16: 104-113.

6. La Torre A, Conca A, Duffy D, Giupponi G, Pompili M, et al. (2013) Sexual dysfunction related to psychotropic drugs: a critical review part II: antipsychotics. Pharmacopsych 46: 201-208.
7. Russell Ravan JP, Thomas N, Paul T, Prasanna S, Thomas N, et al. (2017) The endocrine impact of long term risperidone therapy in Asian Indian patients. Open J Psychiatry Allied Sci 8: 107-112.

8. Ghadirian AM, Chouinard G, Annable L (1982) Sexual dysfunction and plasma prolactin levels in neuroleptic-treated schizophrenic outpatients. J Nerv Ment Dis 170: 463-467.

9. Stimmel GL, Gutierrez MA (2006) Sexual dysfunction and psychotropic medications. CNS Spectr 11: 24-30.

10. Knegtering R, Baselmans P, Castelein S, Bosker F, Bruggeman R, et al. (2005) Predominant role of the 9-hydroxy metabolite of risperidone in elevating blood prolactin levels. Am J Psychiatry 162: 1010-1012.

11. Das D, Talukdar U, Chisty SJS, Das MK, Das S (2015) Serum prolactin level in patients taking olanzapine. Open J Psychiatry Allied Sci 6: 50-58.

12. La Torre A, Giupponi G, Duffy DM, Pompili M, Grözinger M, et al. (2014) Sexual dysfunction related to psychotropic drugs: A critical review. Part III: Mood stabilizers and anxiolytic drugs. Pharmacopsych 47: 1-6. 